\title{
N-LTP: An Open-source Neural Language Technology Platform for Chinese
}

\author{
Wanxiang Che, Yunlong Feng, Libo Qin, Ting Liu \\ Research Center for Social Computing and Information Retrieval \\ Harbin Institute of Technology, China \\ \{car,ylfeng, lbqin, tliu\}@ir.hit.edu.cn
}

\begin{abstract}
We introduce N-LTP, an open-source neural language technology platform supporting six fundamental Chinese NLP tasks: lexical analysis (Chinese word segmentation, part-of-speech tagging, and named entity recognition), syntactic parsing (dependency parsing), and semantic parsing (semantic dependency parsing and semantic role labeling). Unlike the existing state-of-the-art toolkits, such as Stanza, that adopt an independent model for each task, $\mathrm{N}$-LTP adopts the multi-task framework by using a shared pre-trained model, which has the advantage of capturing the shared knowledge across relevant Chinese tasks. In addition, a knowledge distillation method (Clark et al., 2019) where the single-task model teaches the multi-task model is further introduced to encourage the multi-task model to surpass its single-task teacher. Finally, we provide a collection of easy-to-use APIs and a visualization tool to make users to use and view the processing results more easily and directly. To the best of our knowledge, this is the first toolkit to support six Chinese NLP fundamental tasks. Source code, documentation, and pre-trained models are available at https: / / github.com/HIT-SCIR/ltp.
\end{abstract}

\section{Introduction}

There is a wide of range of existing natural language processing (NLP) toolkits such as CoreNLP (Manning et al., 2014), UDPipe (Straka and Straková, 2017), FLAIR (Akbik et al., 2019), spaCy, ${ }^{1}$ and Stanza (Qi et al., 2020) in English, which makes it easier for users to build tools with sophisticated linguistic processing. Recently, the need for Chinese NLP has a dramatic increase in many downstream applications. A Chinese NLP platform usually includes lexical analysis (Chinese word segmentation (CWS), part-of-speech (POS)

\footnotetext{
${ }^{1}$ https://spacy.io
}

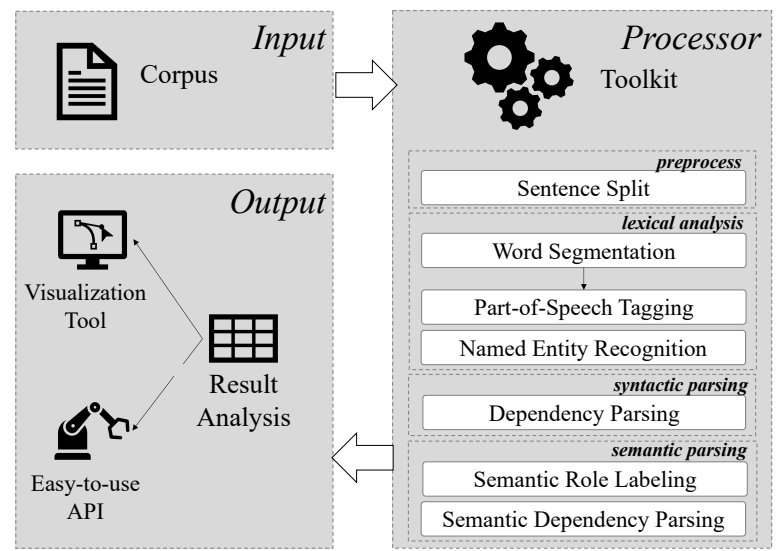

Figure 1: Workflow of the N-LTP. N-LTP takes the Chinese corpus as input and output the analysis results including lexical analysis, syntactic parsing, and semantic parsing. In addition, we provide the visualization tool and easy-to-use API to help users easily use N-LTP.

tagging, and named entity recognition (NER)), syntactic parsing (dependency parsing (DEP)), and semantic parsing (semantic dependency parsing (SDP) and semantic role labeling (SRL)). Unfortunately, there are relatively fewer high-performance and high-efficiency toolkits for Chinese NLP tasks. To fill this gap, it's important to build a Chinese NLP toolkit to support rich Chinese fundamental NLP tasks, and make researchers process NLP tasks in Chinese quickly.

Recently, Qi et al. (2020) introduce the Python NLP toolkit Stanza for multi-lingual languages, including Chinese language. Though Stanza can be directly applied for processing the Chinese texts, it suffers from several limitations. First, it only supports part of Chinese NLP tasks. For example, it fails to handle semantic parsing analysis, resulting in incomplete analysis in Chinese NLP. Second, it trained each task separately, ignoring the shared knowledge across the related tasks, which has been proven effective for Chinese NLP tasks (Qian et al., 2015; Hsieh et al., 2017; Chang et al., 2018). Third, independent modeling method will occupy more 


\begin{tabular}{|c|c|c|c|c|c|}
\hline System & Programming Language & Fully Neural & $\begin{array}{l}\text { State-of-the-art } \\
\text { Performance }\end{array}$ & $\begin{array}{c}\text { Rich Chinese } \\
\text { Fundamental Tasks }\end{array}$ & $\begin{array}{c}\text { Multi-task } \\
\text { Learning }\end{array}$ \\
\hline LTP (Che et al., 2010) & $\mathrm{C}++$ & & & $\sqrt{ }$ & \\
\hline UDPipe (Straka and Straková, 2017) & $\mathrm{C}++$ & & $\sqrt{ }$ & & \\
\hline FLAIR (Akbik et al., 2019) & Python & $\sqrt{ }$ & $\sqrt{ }$ & & \\
\hline Stanza (Qi et al., 2020) & Python & $\sqrt{ }$ & $\sqrt{ }$ & & \\
\hline $\mathrm{N}-\mathrm{LTP}$ & Python & $\sqrt{ }$ & $\sqrt{ }$ & $\sqrt{ }$ & $\sqrt{ }$ \\
\hline
\end{tabular}

Table 1: Feature comparisons of N-LTP against other popular natural language processing toolkits.

memory with the increase of the number of tasks, which makes it hard to deploy for mobile devices in real-word scenario.

To address the aforementioned issues, we introduce N-LTP, a PyTorch-based neural natural language processing toolkit for Chinese NLP, which was built on the SOTA pre-trained model. As shown in Figure 1, given Chinese corpus as input, $\mathrm{N}$-LTP produces comprehensive analysis results, including lexical analysis, syntactic parsing, and semantic parsing. In addition, N-LTP provides easy-to-use APIs and visualization tool, which is user-friendly.

As shown in Table 1, compared to the existing widely-used NLP toolkits, N-LTP has the following advantages:

- Comprehensive Tasks. N-LTP supports rich Chinese fundamental NLP tasks including lexical analysis (word segmentation, part-ofspeech tagging, named entity recognition), syntactic parsing, and semantic parsing (semantic dependency parsing, semantic role labeling). To the best of our knowledge, this is the first neural Chinese toolkit that support six Chinese fundamental NLP tasks.

- Multi-Task Learning. The existing NLP toolkits for the Chinese language all adopt independent models for each task, which ignore the shared knowledge across tasks.

To alleviate this issue, we propose to use the multi-task framework (Collobert et al., 2011) to take advantage of the shared knowledge across all tasks. Meanwhile, multi-task learning with a shared encoder for all six tasks can greatly reduce the occupied memory and improve the speed, which makes N-LTP more efficient, reducing the need for hardware.

In addition, to enable the multi-task learning to enhance each subtask performance, we follow Clark et al. (2019) to adopt the distillation method single-task models teach a multi-task model, helping the multi-task model surpass its all single-task teachers.

- Extensibility. N-LTP works with users' custom modules. Users can easily add a new pre-trained model with a configuration file, in which users can change the pretrained model to any BERT-like model supported by HuggingFace Transformers (Wolf et al., 2019) easily by changing the config. We have made all task training configuration files open-sourced.

- Easy-to-use API and Visualization Tool. N-LTP provides a collection of fundamental APIs, which is convenient for users to use the toolkit without the need for any knowledge. We also provide a visualization tool, which enables users to view the processing results directly. In addition, N-LTP has bindings for many programming languages $(\mathrm{C}++$, Python, Java, Rust, etc.).

- State-of-the-art Performance. We evaluate N-LTP on a total of six Chinese NLP tasks, and find that it achieves state-of-the-art or competitive performance at each task.

N-LTP is fully open-sourced and can support six Chinese fundamental NLP tasks. We hope N-LTP can facilitate Chinese NLP research.

\section{Design and Architecture}

Figure 2 shows an overview of the main architecture of N-LTP. It mainly consists of the components including a shared encoder and different decoders for each task. Our framework shares one encoder for leveraging the shared knowledge across all tasks. Different task decoders are used for each task separately. All tasks are optimized simultaneously via a joint learning scheme. In addition, the knowledge distillation technique is introduced to encourage the multi-task model to surpass its single-task teacher model. 


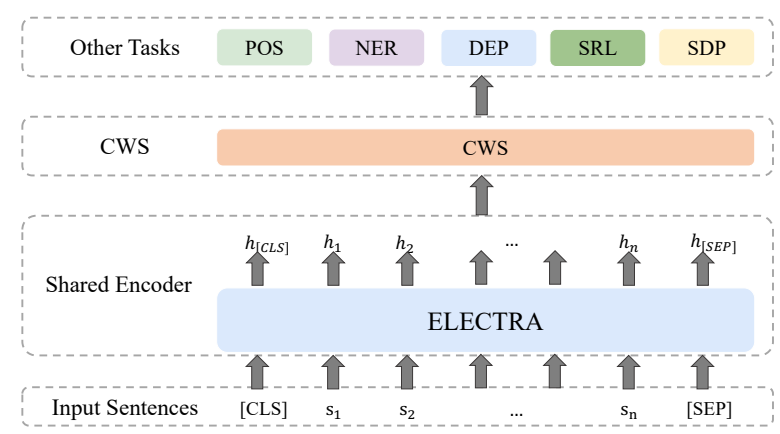

Figure 2: The architecture of the proposed model.

\subsection{Shared Encoder}

Multi-task framework uses a shared encoder to extract the shared knowledge across related tasks, which has obtained remarkable success on various NLP tasks (Qin et al., 2019; Wang et al., 2020; Zhou et al., 2021). Inspired by this, we adopt the SOTA pre-trained model (ELECTRA) (Clark et al., 2020) as the shared encoder to capture shared knowledge across six Chinese tasks.

Given an input utterance $s=\left(s_{1}, s_{2}, \ldots, s_{n}\right)$, we first construct the input sequence by adding specific tokens $s=\left([\mathrm{CLS}], s_{1}, s_{2}, \ldots, s_{n},[\mathrm{SEP}]\right)$, where [CLS] is the special symbol for representing the whole sequence, and [SEP] is the special symbol to separate non-consecutive token sequences (Devlin et al., 2019). ELECTRA takes the constructed input and output the corresponding hidden representations of sequence $\boldsymbol{H}=\left(\boldsymbol{h}_{\text {[CLS] }}\right.$, $\left.\boldsymbol{h}_{1}, \boldsymbol{h}_{2}, \ldots, \boldsymbol{h}_{n}, \boldsymbol{h}_{[\mathrm{SEP}]}\right)$.

\subsection{Chinese Word Segmentation}

Chinese word segmentation (CWS) is a preliminary and important task for Chinese natural language processing (NLP). In N-LTP, following Xue (2003), CWS is regarded as a character based sequence labeling problem.

Specifically, given the hidden representations $\boldsymbol{H}$ $=\left(\boldsymbol{h}_{[\mathrm{CLS}]}, \boldsymbol{h}_{1}, \boldsymbol{h}_{2}, \ldots, \boldsymbol{h}_{n}, \boldsymbol{h}_{[\mathrm{SEP}]}\right)$, we adopt a linear decoder to classify each character:

$$
\boldsymbol{y}_{\boldsymbol{i}}=\operatorname{Softmax}\left(\boldsymbol{W}_{\mathrm{CWS}} \boldsymbol{h}_{\boldsymbol{i}}+\boldsymbol{b}_{\mathrm{CWS}}\right),
$$

where $\boldsymbol{y}_{\boldsymbol{i}}$ denotes the label probability distribution of each character; $\boldsymbol{W}_{\mathrm{CWS}}$ and $\boldsymbol{b}_{\mathrm{CWS}}$ are trainable parameters.

\subsection{POS Tagging}

Part-of-speech (POS) tagging is another fundamental NLP task, which can facilitate the downstream tasks such as syntactic parsing. Following the dominant model in the literature (Ratnaparkhi, 1996;
Huang et al., 2015), POS tagging can be treated as a sequence labeling task.

Similar to CWS, we take the sequence of hidden representations $\boldsymbol{H}$ as input and output the corresponding POS sequence labels, which is formulated as:

$$
\boldsymbol{y}_{\boldsymbol{i}}=\operatorname{Softmax}\left(\boldsymbol{W}_{\mathrm{POS}} \boldsymbol{h}_{\boldsymbol{i}}+\boldsymbol{b}_{\mathrm{POS}}\right),
$$

where $\boldsymbol{y}_{\boldsymbol{i}}$ denotes the POS label probability distribution of the $i$-th character; $\boldsymbol{h}_{\boldsymbol{i}}$ is the first sub-token representation of word $s_{i}$.

\subsection{Named Entity Recognition}

The named entity recognition (NER) is the task of finding the start and end of an entity (people, locations, organizations, etc.) in a sentence and assigning a class for this entity.

Traditional, NER is regarded as a sequence labeling task. After obtaining the hidden representations $\boldsymbol{H}$, we follow Yan et al. (2019a) to adopt the Adapted-Transformer to consider directionand distance-aware characteristic, which can be formulated as:

$$
\hat{\boldsymbol{h}_{\boldsymbol{i}}}=\text { AdaptedTransformer }\left(\boldsymbol{h}_{i}\right),
$$

where $\hat{\boldsymbol{H}}=\left(\hat{\boldsymbol{h}}_{[\mathrm{CLS}]}, \hat{\boldsymbol{h}}_{1}, \hat{\boldsymbol{h}}_{2}, \ldots, \hat{\boldsymbol{h}}_{n}, \hat{\boldsymbol{h}}_{[\mathrm{SEP}]}\right)$ are the updated representations.

Finally, similar to CWS and POS, we use a linear decoder to classify label for each word:

$$
\boldsymbol{y}_{\boldsymbol{i}}=\operatorname{Softmax}\left(\boldsymbol{W}_{\mathrm{NER}} \hat{\boldsymbol{h}}_{i}+\boldsymbol{b}_{\mathrm{NER}}\right),
$$

where $\boldsymbol{y}_{\boldsymbol{i}}$ denotes the NER label probability distribution of each character.

\subsection{Dependency Parsing}

Dependency parsing is the task to analyze the semantic structure of a sentence. In N-LTP, we implement a deep biaffine neural dependency parser (Dozat and Manning, 2017) and einser algorithm (Eisner, 1996) to obtain the parsing result, which is formulated as:

$$
\begin{aligned}
\boldsymbol{r}_{i}^{(\text {head })} & =\operatorname{MLP}^{(\text {head })}\left(\boldsymbol{h}_{i}\right) \\
\boldsymbol{r}_{j}^{(\text {dep })} & =\operatorname{MLP}^{(\text {dep })}\left(\boldsymbol{h}_{j}\right)
\end{aligned}
$$

After obtaining $\boldsymbol{r}_{i}^{(\text {head })}$ and $\boldsymbol{r}_{j}^{(\text {dep})}$, we compute the score for each dependency $i^{\curvearrowleft} j$ by:

$$
\boldsymbol{y}_{i \curvearrowleft j}=\operatorname{BiAffine}\left(\boldsymbol{r}_{i}^{\text {dep }}, \boldsymbol{r}_{j}^{\text {head }}\right) .
$$




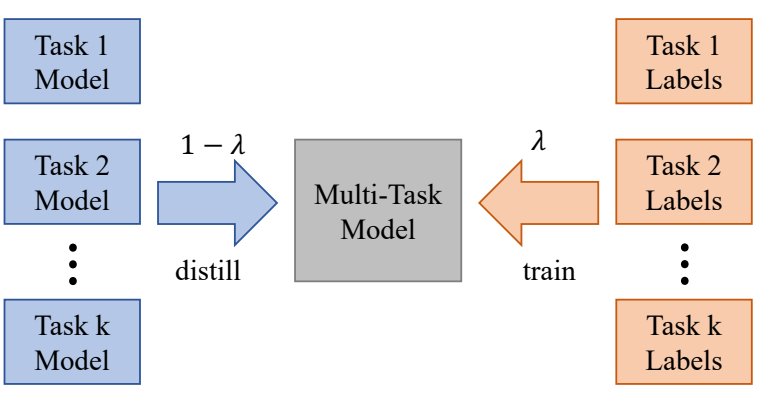

Figure 3: We follow Clark et al. (2019) to adopt the distillation method. This is an overview of the distillation method. $\lambda$ is increased linearly from 0 to 1 over the curriculum of training.

The above process is also used for scoring a labeled dependency $i^{\curvearrowleft} j$, by extending the 1-dim vector $s$ into $L$ dims, where $L$ is the total number of dependency labels.

\subsection{Semantic Dependency Parsing}

Similar to dependency parsing, semantic dependency parsing (Che et al., 2012, SDP) is a task to capture the semantic structure of a sentence. Specifically, given an input sentence, SDP aims at determining all the word pairs related to each other semantically and assigning specific predefined semantic relations. Following Dozat and Manning (2017), we adopt a biaffine module to perform the task, using

$$
\boldsymbol{p}_{i \curvearrowleft j}=\operatorname{sigmoid}\left(\boldsymbol{y}_{i \curvearrowleft j}\right) .
$$

If $\boldsymbol{p}_{i \curvearrowleft j}>0.5$, word $_{i}$ to word $_{j}$ exists an edge.

\subsection{Semantic Role Labeling}

Semantic Role Labeling (SRL) is the task of determining the latent predicate-argument structure of a sentence, which can provide representations to answer basic questions about sentence meaning, including who did what to whom, etc. We adopt an end-to-end SRL model by combining a deep biaffine neural network and a conditional random field (CRF)-based decoder (Cai et al., 2018).

The biaffine module is similar to Section 2.5 and the CRF layer can be formulated as:

$$
P(\hat{y} \mid s)=\frac{\sum_{j=1} \exp f\left(y_{i, j-1}, y_{i, j}, s\right)}{\sum_{y_{i}^{\prime}} \sum_{j=1} \exp f\left(y_{i, j-1}^{\prime}, y_{i, j}^{\prime}, s\right)}
$$

where $\hat{y}$ represents an arbitrary label sequence when predicate is $s_{i}$, and $f\left(y_{i, j-1}, y_{j}, s\right)$ computes the transition score from $y_{i, j-1}$ to $y_{i, j}$.

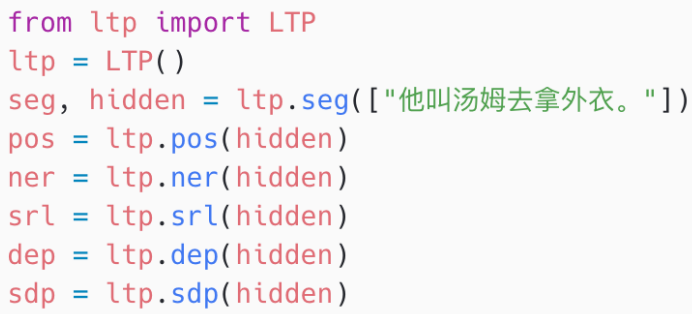

Figure 4: A minimal code snippet.

\subsection{Knowledge Distillation}

When there exist a large number of tasks, it's difficult to ensure that each task task benefits from multi-task learning (Clark et al., 2019).

Therefore, we follow BAM (Clark et al., 2019) to use the knowledge distillation to alleviate this issue, which is shown Figure 3. First, we train each task as the teacher model. Then, N-LTP learns from each trained single-task teacher model while learning from the gold-standard labels simultaneously.

Following BAM (Clark et al., 2019), we adopt teacher annealing distillation algorithm. More specifically, instead of simply shuffling the datasets for our multi-task models, we follow the task sampling procedure from Bowman et al. (2018), where the probability of training on an example for a particular task $\tau$ is proportional to $|D \tau|^{0.75}$. This ensures that tasks with large datasets don't overly dominate the training.

\section{Usage}

$\mathrm{N}$-LTP is a PyTorch-based Chinese NLP toolkit based on the above model. All the configurations can be initialized from JSON files, and thus it is easy for users to use N-LTP where users just need one line of code to load the model or process the input sentences. Specifically, N-LTP can be installed easily by the command:

$\$$ pip install ltp

In addition, $\mathrm{N}-\mathrm{LTP}$ has bindings available for many programming languages, including $\mathrm{C}++$, Python, Java and RUST directly.

\subsection{Easy-to-use API}

We provide rich easy-to-use APIs, which enables users to easily use without the need for any knowledge. The following code snippet in Figure 4 shows 


\begin{tabular}{|c|c|c|c|c|c|c|}
\hline Model & $\begin{array}{c}\text { Chinese Word } \\
\text { Segmentation } \\
F\end{array}$ & $\begin{array}{c}\text { Part-of-Speech } \\
\text { Tagging } \\
F_{L A S}\end{array}$ & $\begin{array}{c}\text { Named Entity } \\
\text { Recognition } \\
F\end{array}$ & $\begin{array}{c}\text { Dependency } \\
\text { Parsing } \\
F\end{array}$ & $\begin{array}{c}\text { Semantic Dependency } \\
\text { Parsing } \\
F\end{array}$ & $\begin{array}{c}\text { Semantic Role } \\
\text { Labeling } \\
F\end{array}$ \\
\hline Stanza (Qi et al., 2020) & 92.40 & 98.10 & 89.50 & 84.98 & - & - \\
\hline $\mathrm{N}-\mathrm{LTP}$ trained separately & 98.55 & 98.35 & 95.41 & 90.12 & 74.47 & 79.23 \\
\hline $\mathrm{N}$-LTP trained jointly with distillation & 99.18 & 98.69 & 95.97 & 90.19 & 76.62 & 79.49 \\
\hline
\end{tabular}

Table 2: Main Results. "-" represents the absence of tasks in the Stanza toolkit and we cannot report the results.

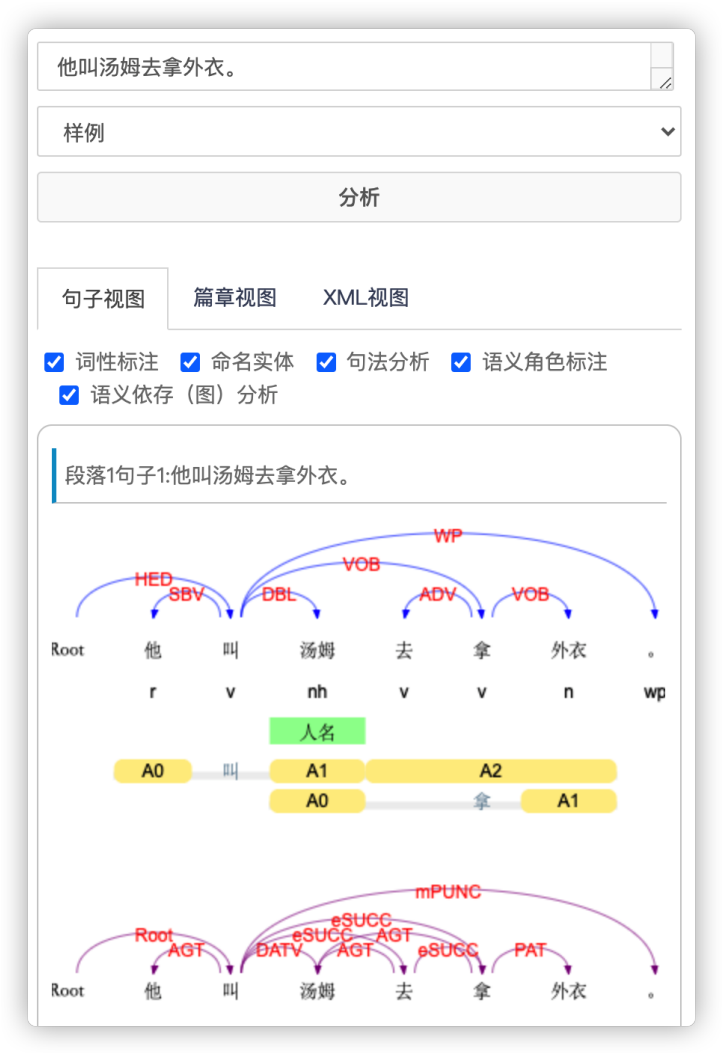

Figure 5: LTP annotates a Chinese sentence “他叫汤姆 去拿外衣。/ He told Tom to get his coat.”. The output is visualized by our visualization demo.

a minimal usage of N-LTP for downloading models, annotating a sentence with customized models, and predicting all annotations.

\subsection{Visualization Tool}

In addition, a visualization tool is proposed for users to view the processing results directly. Specifically, we build an interactive web demo that runs the pipeline interactively, which is publicly available at http://ltp.ai/demo.html. The visualization tool is shown in Figure 5.

\section{Experiments}

\subsection{Experimental Setting}

To evaluate the efficiency of our multi-task model, we conduct experiments on six Chinese tasks.
The N-LTP model is based on the Chinese ELECTRA base (Cui et al., 2020). The learning ratio (lr) for teacher models, student model and CRF layer is $\{1 e-4\},\{1 e-4\},\{1 e-3\}$, respectively. The gradient clip value adopted in our experiment is 1.0 and the warmup proportion is 0.02. We use BertAdam (Devlin et al., 2019) to optimize the parameters and adopted the suggested hyper-parameters for optimization.

\subsection{Results}

We compare N-LTP with the state-of-the-art toolkit Stanza. For a fair comparison, we conduct experiments on the same datasets that Stanza adopted.

The results are shown in Table 2, we have the following observations:

- N-LTP outperforms Stanza on four common tasks including CWS, POS, NER, and DEP by a large margin, which shows the superiority of our proposed toolkit.

- The multi-task learning outperforms the model with independently trained. This is because that the multi-task framework can consider the shared knowledge which can promote each task compared with the independently training paradigm.

\subsection{Analysis}

\subsubsection{Speedup and Memory Reduction}

In this section, we perform the speed and memory test on the Tesla V100-SXM2-16GB and all models were speed-tested on the 10,000 sentences of the People's Daily corpus with a batch size of 8 . In all experiments, N-LTP performs six tasks (CWS, POS, NER, DEP, SDP, SRL) while St an za only conduct four tasks (CWS, POS, NER, DEP).

\footnotetext{
${ }^{2} 10$ corpus for training CWS task includes PKU, MSR, AS, CITYU, XU, CTB, UDC, CNC, WTB and ZX.

${ }^{3}$ http://ir.hit.edu.cn/sdp2020ccl
} 


\begin{tabular}{lrrrrrr}
\hline Task & Model & Dataset & Metric & $\begin{array}{c}\text { State-of-the-art } \\
\text { Performance }\end{array}$ & $\begin{array}{c}\text { N-LTP } \\
\text { trained separately }\end{array}$ & $\begin{array}{c}\text { N-LTP } \\
\text { trained jointly }\end{array}$ \\
\hline CWS & BERT (Huang et al., 2019) & 10 Corpus ${ }^{2}$ & F1 & 97.10 & 97.42 & $\mathbf{9 7 . 5 0}$ \\
POS & Glyce+BERT (Meng et al., 2019) & CTB9 & F1 & 93.15 & 94.57 & $\mathbf{9 5 . 1 7}$ \\
NER & ZEN (Diao et al., 2020) & MSRA & F1 & 95.25 & 94.95 & $\mathbf{9 5 . 7 8}$ \\
NER & DGLSTM-CRF (Jie and Lu, 2019) & OntoNotes & F1 & 79.92 & 84.08 & $\mathbf{8 4 . 3 8}$ \\
SRL & BiLSTM-Span (Ouchi et al., 2018) & CONLL12 & F1 & 75.75 & 78.20 & $\mathbf{8 1 . 6 5}$ \\
DEP & Joint-Multi-BERT (Yan et al., 2019b) & CTB9 & F1 1 $12 A S$ & 81.71 & 81.69 & $\mathbf{8 4 . 0 3}$ \\
SDP & SuPar $^{3}$ & CCL2020 & F1 $1_{L A S}$ & $\mathbf{8 0 . 3 8}$ & 76.27 & 75.76 \\
\hline
\end{tabular}

Table 3: The results of N-LTP comparation to other state-of-the-art performance..

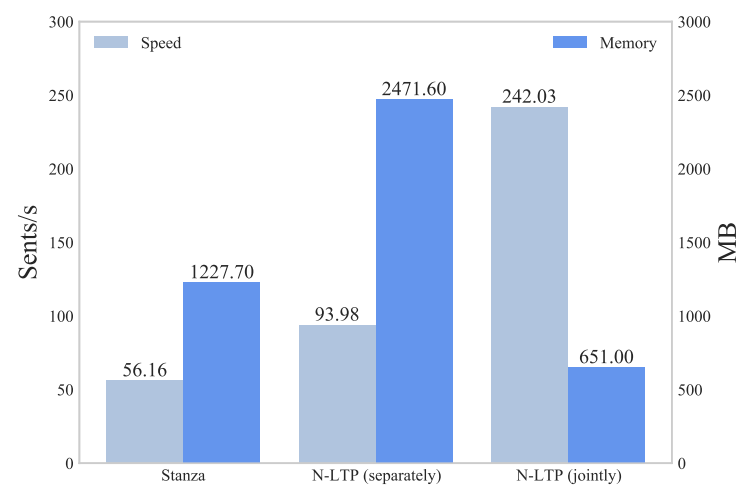

Figure 6: Speed and Memory test for N-LTP.

Speedup We compare the speed between Stanza, N-LTP-separately and N-LTP-jointly and the results are shown in Figure 6. From the results of speed test, we have two interesting observations: (1) N-LTP trained separately achieves the $\mathrm{x} 1.7$ speedup compared with Stanza. We attribute that N-LTP adopts the transformer as an encoder that can be calculated in parallel while Stanza uses LSTM which can only process sentences word by word; (2) N-LTP trained jointly with distillation obtains the $\mathrm{x} 4.3$ speedup compared with separate modeling paradigm. This is because that our model utilizes the multi-task to perform all tasks while the independent models can be only processed all tasks in a pipeline mode.

Memory Reduction For memory test, we have the following observation: (1) N-LTP trained separately occupy more memory than Stanza. This is because N-LTP performs six tasks while Stanza only conduct four tasks. (2) Though performing six tasks, N-LTP trained jointly only requires half the memory compared to Stanza. We attribute it to the fact that the multi-task framework with a shared encoder can greatly reduce the running memory.

\subsubsection{Comparation with Other SOTA Single Models}

To further verify the effectiveness of N-LTP, we compare our framework with the existing state-ofthe-art single models on six Chinese fundamental tasks. In this comparison, we conduct experiments on the same wildly-used dataset in each task for a fair comparison. In addition, we use BERT rather than ELECTRA as the shared encoder, because the prior work adopts BERT.

Table 3 shows the results, we observe that our framework obtains best performance on five out of six tasks including CWS, POS, NER, SRL, and DEP, which demonstrates the effectiveness of our framework. On the SDP task, N-LTP underperforms the best baseline. This is because many tricks are used in the prior model for SDP task and we just use the basic multi-task framework.

\section{Conclusion}

In this paper, we presented N-LTP, an open-source neural language technology platform supporting Chinese. To the best of our knowledge, this is the first Chinese toolkit that supports six fundamental Chinese NLP tasks. Experimental results show $\mathrm{N}$-LTP obtains state-of-the-art or competitive performance and has high speed. We hope N-LTP can facilitate Chinese NLP research.

\section{Acknowledgements}

We thank the anonymous reviewers for their detailed and constructive comments. The first three authors contributed equally. Wanxiang Che is the corresponding author. This work was supported by the National Key R\&D Program of China via grant 2020AAA0106501 and the National Natural Science Foundation of China (NSFC) via grant 61976072 and 61772153. Libo is also supported by the Zhejiang Lab's International Talent Fund for Young Professionals. 


\section{References}

Alan Akbik, Tanja Bergmann, Duncan Blythe, Kashif Rasul, Stefan Schweter, and Roland Vollgraf. 2019. FLAIR: An easy-to-use framework for state-of-theart NLP. In Proceedings of the 2019 Conference of the North American Chapter of the Association for Computational Linguistics (Demonstrations), pages 54-59, Minneapolis, Minnesota. Association for Computational Linguistics.

Samuel R. Bowman, Ellie Pavlick, Edouard Grave, Benjamin Van Durme, Alex Wang, Jan Hula, Patrick Xia, Raghavendra Pappagari, R. Thomas McCoy, Roma Patel, Najoung Kim, Ian Tenney, Yinghui Huang, Katherin Yu, Shuning Jin, and Berlin Chen. 2018. Looking for elmo's friends: Sentence-level pretraining beyond language modeling. CoRR, $\mathrm{abs} / 1812.10860$.

Jiaxun Cai, Shexia He, Zuchao Li, and Hai Zhao. 2018. A full end-to-end semantic role labeler, syntacticagnostic over syntactic-aware? In Proceedings of the 27th International Conference on Computational Linguistics, pages 2753-2765, Santa Fe, New Mexico, USA. Association for Computational Linguistics.

Yung-Chun Chang, Fang Yi Lee, and Chun Hung Chen. 2018. A public opinion keyword vector for social sentiment analysis research. In 2018 Tenth International Conference on Advanced Computational Intelligence (ICACI), pages 752-757. IEEE.

Wanxiang Che, Zhenghua Li, and Ting Liu. 2010. LTP: A Chinese language technology platform. In Coling 2010: Demonstrations, pages 13-16, Beijing, China. Coling 2010 Organizing Committee.

Wanxiang Che, Meishan Zhang, Yanqiu Shao, and Ting Liu. 2012. SemEval-2012 task 5: Chinese semantic dependency parsing. In *SEM 2012: The First Joint Conference on Lexical and Computational Semantics - Volume 1: Proceedings of the main conference and the shared task, and Volume 2: Proceedings of the Sixth International Workshop on Semantic Evaluation (SemEval 2012), pages 378-384, Montréal, Canada. Association for Computational Linguistics.

Kevin Clark, Minh-Thang Luong, Urvashi Khandelwal, Christopher D. Manning, and Quoc V. Le. 2019. BAM! born-again multi-task networks for natural language understanding. In Proceedings of the 57th Annual Meeting of the Association for Computational Linguistics, pages 5931-5937, Florence, Italy. Association for Computational Linguistics.

Kevin Clark, Minh-Thang Luong, Quoc V. Le, and Christopher D. Manning. 2020. ELECTRA: pretraining text encoders as discriminators rather than generators. In 8th International Conference on Learning Representations, ICLR 2020, Addis Ababa, Ethiopia, April 26-30, 2020. OpenReview.net.

Ronan Collobert, Jason Weston, Léon Bottou, Michael Karlen, Koray Kavukcuoglu, and Pavel Kuksa. 2011. Natural language processing (almost) from scratch. Journal of machine learning research, 12(ARTICLE):2493-2537.

Yiming Cui, Wanxiang Che, Ting Liu, Bing Qin, Shijin Wang, and Guoping Hu. 2020. Revisiting pre-trained models for Chinese natural language processing. In Findings of the Association for Computational Linguistics: EMNLP 2020, pages 657-668, Online. Association for Computational Linguistics.

Jacob Devlin, Ming-Wei Chang, Kenton Lee, and Kristina Toutanova. 2019. BERT: Pre-training of deep bidirectional transformers for language understanding. In Proceedings of the 2019 Conference of the North American Chapter of the Association for Computational Linguistics: Human Language Technologies, Volume 1 (Long and Short Papers), pages 4171-4186, Minneapolis, Minnesota. Association for Computational Linguistics.

Shizhe Diao, Jiaxin Bai, Yan Song, Tong Zhang, and Yonggang Wang. 2020. ZEN: Pre-training Chinese text encoder enhanced by n-gram representations. In Findings of the Association for Computational Linguistics: EMNLP 2020, pages 4729-4740, Online. Association for Computational Linguistics.

Timothy Dozat and Christopher D. Manning. 2017. Deep biaffine attention for neural dependency parsing. In 5th International Conference on Learning Representations, ICLR 2017, Toulon, France, April 24-26, 2017, Conference Track Proceedings. OpenReview.net.

Jason M. Eisner. 1996. Three new probabilistic models for dependency parsing: An exploration. In COLING 1996 Volume 1: The 16th International Conference on Computational Linguistics.

Yu-Lun Hsieh, Yung-Chun Chang, Yi-Jie Huang, ShuHao Yeh, Chun-Hung Chen, and Wen-Lian Hsu. 2017. MONPA: Multi-objective named-entity and part-of-speech annotator for Chinese using recurrent neural network. In Proceedings of the Eighth International Joint Conference on Natural Language Processing (Volume 2: Short Papers), pages 80-85, Taipei, Taiwan. Asian Federation of Natural Language Processing.

Weipeng Huang, Xingyi Cheng, Kunlong Chen, Taifeng Wang, and Wei Chu. 2019. Toward fast and accurate neural chinese word segmentation with multi-criteria learning. CoRR, abs/1903.04190.

Zhiheng Huang, Wei Xu, and Kai Yu. 2015. Bidirectional LSTM-CRF models for sequence tagging. CoRR, abs/1508.01991.

Zhanming Jie and Wei Lu. 2019. Dependency-guided LSTM-CRF for named entity recognition. In Proceedings of the 2019 Conference on Empirical Methods in Natural Language Processing and the 9th International Joint Conference on Natural Language Processing (EMNLP-IJCNLP), pages 3862-3872, Hong Kong, China. Association for Computational Linguistics. 
Christopher Manning, Mihai Surdeanu, John Bauer, Jenny Finkel, Steven Bethard, and David McClosky. 2014. The Stanford CoreNLP natural language processing toolkit. In Proceedings of 52nd Annual Meeting of the Association for Computational Linguistics: System Demonstrations, pages 55-60, Baltimore, Maryland. Association for Computational Linguistics.

Yuxian Meng, Wei Wu, Fei Wang, Xiaoya Li, Ping Nie, Fan Yin, Muyu Li, Qinghong Han, Xiaofei Sun, and Jiwei Li. 2019. Glyce: Glyph-vectors for chinese character representations. In Advances in Neural Information Processing Systems 32: Annual Conference on Neural Information Processing Systems 2019, NeurIPS 2019, December 8-14, 2019, Vancouver, BC, Canada, pages 2742-2753.

Hiroki Ouchi, Hiroyuki Shindo, and Yuji Matsumoto. 2018. A span selection model for semantic role labeling. In Proceedings of the 2018 Conference on Empirical Methods in Natural Language Processing, pages 1630-1642, Brussels, Belgium. Association for Computational Linguistics.

Peng Qi, Yuhao Zhang, Yuhui Zhang, Jason Bolton, and Christopher D. Manning. 2020. Stanza: A python natural language processing toolkit for many human languages. In Proceedings of the 58th Annual Meeting of the Association for Computational Linguistics: System Demonstrations, pages 101-108, Online. Association for Computational Linguistics.

Tao Qian, Yue Zhang, Meishan Zhang, Yafeng Ren, and Donghong Ji. 2015. A transition-based model for joint segmentation, POS-tagging and normalization. In Proceedings of the 2015 Conference on Empirical Methods in Natural Language Processing, pages 1837-1846, Lisbon, Portugal. Association for Computational Linguistics.

Libo Qin, Wanxiang Che, Yangming Li, Haoyang Wen, and Ting Liu. 2019. A stack-propagation framework with token-level intent detection for spoken language understanding. In Proceedings of the 2019 Conference on Empirical Methods in Natural Language Processing and the 9th International Joint Conference on Natural Language Processing (EMNLP-IJCNLP), pages 2078-2087, Hong Kong, China. Association for Computational Linguistics.

Adwait Ratnaparkhi. 1996. A maximum entropy model for part-of-speech tagging. In Conference on Empirical Methods in Natural Language Processing.

Milan Straka and Jana Straková. 2017. Tokenizing, POS tagging, lemmatizing and parsing UD 2.0 with UDPipe. In Proceedings of the CoNLL 2017 Shared Task: Multilingual Parsing from Raw Text to Universal Dependencies, pages 88-99, Vancouver, Canada. Association for Computational Linguistics.

Yiren Wang, ChengXiang Zhai, and Hany Hassan. 2020 Multi-task learning for multilingual neural machine translation. In Proceedings of the 2020 Conference on Empirical Methods in Natural Language Processing (EMNLP), pages 1022-1034, Online. Association for Computational Linguistics.

Thomas Wolf, Lysandre Debut, Victor Sanh, Julien Chaumond, Clement Delangue, Anthony Moi, Pierric Cistac, Tim Rault, Rémi Louf, Morgan Funtowicz, Joe Davison, Sam Shleifer, Patrick von Platen, Clara Ma, Yacine Jernite, Julien Plu, Canwen Xu, Teven Le Scao, Sylvain Gugger, Mariama Drame, Quentin Lhoest, and Alexander M. Rush. 2019. Huggingface's transformers: State-of-the-art natural language processing. ArXiv, abs/1910.03771.

Nianwen Xue. 2003. Chinese word segmentation as character tagging. In International Journal of Computational Linguistics \& Chinese Language Processing, Volume 8, Number 1, February 2003: Special Issue on Word Formation and Chinese Language Processing, pages 29-48.

Hang Yan, Bocao Deng, Xiaonan Li, and Xipeng Qiu. 2019a. Tener: Adapting transformer encoder for named entity recognition.

Hang Yan, Xipeng Qiu, and Xuanjing Huang. 2019b. A unified model for joint chinese word segmentation and dependency parsing. CoRR, abs/1904.04697.

Baohang Zhou, Xiangrui Cai, Ying Zhang, and Xiaojie Yuan. 2021. An end-to-end progressive multi-task learning framework for medical named entity recognition and normalization. In Proceedings of the 59th Annual Meeting of the Association for Computational Linguistics and the 11th International Joint Conference on Natural Language Processing (Volume 1: Long Papers), pages 6214-6224, Online. Association for Computational Linguistics. 